\title{
Stability of Nonlinear Fractional Neutral Differential Difference Systems
}

\author{
Kewei Liu ${ }^{1,2}$ and Wei Jiang ${ }^{2}$ \\ ${ }^{1}$ School of Mathematics, Hefei University of Technology, Hefei 230009, China \\ ${ }^{2}$ School of Mathematical Sciences, Anhui University, Hefei 230039, China \\ Correspondence should be addressed to Kewei Liu; kweiliu@163.com
}

Received 30 October 2013; Accepted 29 June 2014; Published 14 July 2014

Academic Editor: Zhen Jin

Copyright (c) $2014 \mathrm{~K}$. Liu and W. Jiang. This is an open access article distributed under the Creative Commons Attribution License, which permits unrestricted use, distribution, and reproduction in any medium, provided the original work is properly cited.

\begin{abstract}
We study the stability of a class of nonlinear fractional neutral differential difference systems equipped with the Caputo derivative. We extend Lyapunov-Krasovskii theorem for the nonlinear fractional neutral systems. Conditions of stability and instability are obtained for the nonlinear fractional neutral systems.
\end{abstract}

\section{Introduction}

Recently, fractional differential equations have attracted great attention. It has been proved that fractional differential equations are valuable tools in the modeling of many phenomena in various fields of engineering, physics, and economics. For details and examples, see [1-5] and the references therein.

Stability analysis is always one of the most important issues in the theory of differential equations and their applications for both deterministic and stochastic cases. Recently, stability of fractional differential equations has attracted increasing interest. Since fractional derivatives are nonlocal and have weakly singular kernels, the analysis on stability of fractional differential equations is more complex than that of classical differential equations. The earliest study on stability of fractional differential equations started in [6]; the author studied the case of linear Caputo fractional differential equations. Since then, many researchers have done further studies on the stability of fractional differential systems [7-18]. For more details about the stability results and the methods available to analyze the stability of fractional differential systems, the reader may refer to the recent survey papers $[19,20]$ and the references therein.

As we all know, Lyapunov's second method provides a way to analyze the stability of a system without explicitly solving the differential equations. It is necessary to extend Lyapunov's second method to fractional systems. In $[13,14]$, the fractional
Lyapunov's second method was proposed, and the authors extended the exponential stability of integer order differential system to the Mittag-Leffler stability of fractional differential system. In [15], by using Bihari's and Bellman-Gronwall's inequality, an extension of Lyapunov's second method for fractional-order systems was proposed. In [16-18], Baleanu et al. extended Lyapunov's method to fractional functional differential systems and developed the Lyapunov-Krasovskii stability theorem, Lyapunov-Razumikhin stability theorem, and Mittag-Leffler stability theorem for fractional functional differential systems.

As far as we know, there are few papers with respect to the stability of fractional neutral systems. In this paper, we consider the stability of a class of nonlinear fractional neutral differential difference equations with the Caputo derivative. Motivated by Li et al. [13, 14], Baleanu et al. [16], and Cruz and Hale [21], we address the stability of fractional neutral systems. Specifically, we extend the Lyapunov-Krasovskii method for the nonlinear fractional neutral differential difference systems.

The rest of the paper is organized as follows. In Section 2, we give some notations and recall some concepts and preparation results. In Section 3, we develop the LyapunovKrasovskii theorem for the nonlinear fractional neutral differential difference systems; results of stability, asymptotically stability, uniform stability, uniform asymptotically stability, and instability for the nonlinear fractional neutral systems are 
presented. Section 4 brings an example to illustrate the results presented and finally Section 5 concludes the work.

\section{Preliminaries}

In this section, we introduce notations, definitions, and preliminary facts needed here. Throughout this paper, let $\mathbb{R}^{n}$ be a real $n$-dimensional linear vector space with the norm | $\mid$; let $\mathscr{C}=C\left([-r, 0], \mathbb{R}^{n}\right)$ be the space of continuous functions taking $[-r, 0]$ into $\mathbb{R}^{n}$ with $\|\phi\|, \phi \in \mathscr{C}$ defined by $\|\phi\|=\sup _{s \in[-r, 0]}|\phi(s)|, \mathscr{C}(M)=\{\phi \in \mathscr{C}:\|\phi\| \leq M\}$, $r>0, M>0$ be constants. Let $A C([a, b], \mathbb{R})$ be the space of functions $f(t)$ which are absolutely continuous on $[a, b]$ and let $A C^{n}([a, b], \mathbb{R})$ be the space of functions $f(t)$ which have continuous derivatives up to order $n-1$ on $[a, b]$ such that $f^{(n-1)}(t) \in A C([a, b], \mathbb{R})$. If $\sigma \in \mathbb{R}, A>0$ and $x \in$ $C\left([\sigma-r, \sigma+A], \mathbb{R}^{n}\right)$, then, for any $t \in[\sigma, \sigma+A]$, we let $x_{t} \in \mathscr{C}$ be defined by $x_{t}(\theta)=x(t+\theta), \theta \in[-r, 0]$.

Let us recall the following definitions. For more details, we refer the reader to [1-5].

Definition 1. The fractional order integral of a function $f$ : $\left[t_{0}, \infty\right) \rightarrow \mathbb{R}$ of order $\alpha \in \mathbb{R}^{+}=[0,+\infty)$ is defined by

$$
I_{t_{0}}^{\alpha} f(t)=\frac{1}{\Gamma(\alpha)} \int_{t_{0}}^{t}(t-s)^{\alpha-1} f(s) d s,
$$

where $\Gamma(\cdot)$ is the gamma function.

Definition 2. For a function $f$ given on the interval $\left[t_{0}, \infty\right)$, the $\alpha$ order Riemann-Liouville fractional derivative of $f$ is defined by

$$
\begin{array}{r}
D_{t_{0}}^{\alpha} f(t)=\frac{1}{\Gamma(n-\alpha)}\left(\frac{d}{d t}\right)^{n} \int_{t_{0}}^{t}(t-s)^{n-\alpha-1} f(s) d s, \\
n-1<\alpha<n, \quad n \in \mathbb{N},
\end{array}
$$

where $\mathbb{N}=\{1,2,3, \ldots\}$.

Definition 3. For a function $f \in A C^{n}\left(\left[t_{0}, b\right], \mathbb{R}\right)$, the $\alpha$ order Caputo fractional derivative of $f$ is defined by

$$
\begin{array}{r}
{ }^{c} D_{t_{0}}^{\alpha} f(t)=\frac{1}{\Gamma(n-\alpha)} \int_{t_{0}}^{t}(t-s)^{n-\alpha-1} f^{(n)}(s) d s, \\
n-1<\alpha<n, \quad n \in \mathbb{N} .
\end{array}
$$

Some properties of the aforementioned operators are recalled as follows $[1,5]$.

Property 4. The following results are especially interesting.

(i) For $v>-1$, we have $D_{t_{0}}^{\alpha}\left(t-t_{0}\right)^{v}=(\Gamma(1+v) / \Gamma(1+\nu-$ $\alpha))\left(t-t_{0}\right)^{\nu-\alpha}$.

(ii) When $0<\alpha<1$, we have

$$
{ }^{c} D_{t_{0}}^{\alpha} f(t)=D_{t_{0}}^{\alpha} f(t)-\frac{f\left(t_{0}\right)\left(t-t_{0}\right)^{-\alpha}}{\Gamma(1-\alpha)} .
$$

(iii) For $\alpha \in(0,1), T \geq t_{0}$, and $f \in C\left(\left[t_{0}, T\right], \mathbb{R}^{n}\right)$, we have ${ }^{c} D_{t_{0}}^{\alpha} I_{t_{0}}^{\alpha} f(t)=f(t), I_{t_{0}}^{\alpha c} D_{t_{0}}^{\alpha} f(t)=f(t)-f\left(t_{0}\right)$.
Remark 5. From Property 4 , if ${ }^{c} D_{t_{0}}^{\alpha} f(t) \geq 0, \alpha \in(0,1)$, then, for $t \geq t_{0}$, we have the following.

(i) $f(t) \geq f\left(t_{0}\right)$.

(ii) In general, it is not true that $f(t)$ is nondecreasing in $t$.

In [21], Cruz and Hale studied a class of functional difference operators which are very useful in stability theory and the asymptotic behavior of solutions of functional differential equations of neutral type. Suppose $\tau \in \mathbb{R}, g:[\tau,+\infty) \times \mathscr{C} \rightarrow$ $\mathbb{R}^{n}$ is continuous, $g(t, \phi)$ is linear in $\phi$, and there are an $n \times n$ matrix $\mu(t, \theta), t \in[\tau,+\infty), \theta \in[-r, 0]$, of bounded variation in $\theta$ and a scalar function $l(s)$ continuously nondecreasing for $s \in[0, r], l(0)=0$, such that

$$
\begin{gathered}
g(t, \phi)=\int_{-r}^{0}\left[d_{\theta} \mu(t, \theta)\right] \phi(\theta), \\
\left|\int_{-s}^{0}\left[d_{\theta} \mu(t, \theta)\right] \phi(\theta)\right| \leq l(s) \sup _{\theta \in[-s, 0]}|\phi(\theta)|,
\end{gathered}
$$

for all $t \in[\tau,+\infty), \phi \in \mathscr{C}$. Define the linear functional difference operator

$$
\mathscr{D}: \mathbb{R} \times \mathscr{C} \longrightarrow \mathbb{R}^{n}
$$

by

$$
\mathscr{D}(t, \phi)=\phi(0)-g(t, \phi) .
$$

For any $H \in C\left([\tau,+\infty), \mathbb{R}^{n}\right)$, the space of continuous functions taking $[\tau,+\infty)$ into $\mathbb{R}^{n}, \sigma \in[\tau,+\infty)$ consider the equation

$$
\begin{array}{r}
\mathscr{D}\left(t, x_{t}\right)=\mathscr{D}(\sigma, \varphi)+H(t)-H(\sigma), \\
t \geq \sigma, \quad x_{\sigma}=\varphi, \quad \varphi \in \mathscr{C} .
\end{array}
$$

Definition 6 (see [21]). Suppose $\mathscr{H}$ is a subset of $C\left([\tau,+\infty), \mathbb{R}^{n}\right)$. Ones says the operator $\mathscr{D}$ is uniformly stable with respect to $\mathscr{H}$ if there are constants $K, M$ such that, for any $\varphi \in \mathscr{C}, \sigma \in[\tau,+\infty)$ and $\mathscr{H}$, the solution $x(t, \sigma, \varphi, H)$ of (8) satisfies

$$
x_{t}(\sigma, \varphi, H) \leq K|\varphi|+M \sup _{u \in[\sigma, t]}|H(u)-H(\sigma)|, \quad t \geq \sigma .
$$

Lemma 7 (see [21]). If $\mathscr{D}$ is uniformly stable with respect to $C\left([\tau,+\infty), \mathbb{R}^{n}\right)$, then there are positive constants $a, b, c, d$ such that, for any $h \in C\left([\tau,+\infty), \mathbb{R}^{n}\right), \sigma \in[\tau,+\infty)$, the solution $x(t, \sigma, \varphi, h)$ of the equation

$$
\mathscr{D}\left(t, x_{t}\right)=h(t), \quad t \geq \sigma, x_{\sigma}=\varphi,
$$

satisfies

$$
\begin{aligned}
\left\|x_{t}(\sigma, \varphi, h)\right\| \leq & e^{-a(t-\sigma)}\left(b\|\varphi\|+c \sup _{u \in[\sigma, t]}|h(u)|\right) \\
& +d \sup _{u \in[\sigma, t]}|h(u)|,
\end{aligned}
$$


for all $t \geq \sigma$. Furthermore, the constants $a, b, c, d$ can be chosen so that for any $s \in[\sigma,+\infty)$

$$
\begin{gathered}
\left\|x_{t}(\sigma, \varphi, h)\right\| \leq e^{-a(t-s)}\left(b\|\varphi\|+c \sup _{u \in[\sigma, t]}|h(u)|\right) \\
+d \sup _{u \in[s, t]}|h(u)|,
\end{gathered}
$$

for all $t \geq s+r$.

Definition 8 (see [22]). Suppose $\mathscr{D}: \mathscr{C} \rightarrow \mathbb{R}^{n}$ is linear, continuous, and atomic at 0 and let $\mathscr{C}_{\mathscr{D}}=\{\phi \in \mathscr{C}: \mathscr{D}(\phi)=$ $0\}$. The operator $\mathscr{D}$ is said to be stable if the zero solution of the homogeneous difference equation,

$$
\mathscr{D}\left(y_{t}\right)=0, \quad t \geq 0, y_{0}=\varphi \in \mathscr{C}_{\mathscr{D}},
$$

is uniformly asymptotically stable.

Definition 9 (see [23]). The matrix $B \in \mathbb{R}^{n \times n}$ is Schur stable if the spectrum of the matrix lies in the open unit disc of the complex plane.

Consider a simple operator $\mathscr{D}: \mathscr{D}\left(x_{t}\right)=x(t)-B x(t-r)$, where the matrix $B$ is Schur stable. Let $x(t)$ be a solution of the homogeneous difference equation

$$
\mathscr{D}\left(x_{t}\right)=0, \quad t \geq 0, x_{0}=\varphi \in \mathscr{C}_{\mathscr{D}} .
$$

Then, for a given $t \geq 0$, there must exist a positive integer number $k$ such that $t \in[(k-1) r, k r]$. It follows from (14) that

$$
x(t)=B^{k} x(t-k r) .
$$

Since the matrix $B$ is Schur stable, there exist $L \geq 1$ and $0<$ $\zeta<1$ such that the inequality $\left\|B^{k}\right\|_{0} \leq L \zeta^{k}$ holds. Therefore we have

$$
|x(t)|=\left|B^{k} x(t-k r)\right| \leq\left\|B^{k}\right\|_{0}|x(t-k r)| \leq L \zeta^{k}\|\varphi\| .
$$

Since $\lim _{k \rightarrow \infty} \zeta^{k}=0$, it follows from (16) that the zero solution of the homogeneous difference equation (14) is uniformly asymptotically stable. Therefore we have the following remark.

Remark 10. Let $\mathscr{D}\left(t, x_{t}\right)=x(t)-B x(t-r)$, where $B$ is Schur stable. Then $\mathscr{D}$ is stable.

\section{Main Results}

In this section, we consider the stability of the following nonlinear fractional neutral differential difference system:

$$
{ }^{c} D_{t_{0}}^{\alpha}\left[\mathscr{D}\left(t, x_{t}\right)\right]=f\left(t, x_{t}\right), \quad t>t_{0},
$$

with the initial condition

$$
x_{t_{0}}=\varphi,
$$

where $0<\alpha<1, f: \mathbb{R} \times \mathscr{C} \rightarrow \mathbb{R}^{n}$ is continuous, Lipschitz in $x_{t}$, and takes closed bounded sets into bounded sets and $g(t, 0)=f(t, 0) \equiv 0$; the linear difference operator $\mathscr{D}$ is defined in (7) with $g$ satisfying (5); that is, $g(t, \phi)$ is uniformly nonatomic at zero [22]. Here, we always assume that fractional order neutral system (17) with initial condition (18) has a unique continuous solution $x(t)$ which depends continuously upon $t_{0}, \varphi$.

If $V: \mathbb{R} \times \mathscr{C} \rightarrow \mathbb{R}$ is continuously differentiable, we define the Caputo fractional derivative ${ }^{c} D_{t_{0}}^{\gamma} V(t, \phi)$ along the solution $x_{t}=x_{t}\left(t_{0}, \varphi\right)$ of (17)-(18) as

$$
\begin{array}{r}
{ }^{c} D_{t_{0}}^{\gamma} V\left(t, x_{t}\right)=\frac{1}{\Gamma(1-\gamma)} \int_{t_{0}}^{t}(t-s)^{-\gamma} V^{\prime}\left(s, x_{s}\right) d s, \\
0<\gamma<1 .
\end{array}
$$

Definition 11. We say that the zero solution $x=0$ of (17) is stable if, for any $t_{0} \in \mathbb{R}$ and any $\varepsilon>0$, there exists a $\delta=$ $\delta\left(t_{0}, \varepsilon\right)$ such that any solution $x(t)=x\left(t, t_{0}, \varphi\right)$ of (17) with initial value $\varphi$ at $t_{0},\|\varphi\|<\delta$, satisfies $|x(t)|<\varepsilon$ for $t \geq t_{0}$. It is asymptotically stable if it is stable and, for any $t_{0} \in \mathbb{R}$ and any $\varepsilon>0$, there exists a $\delta_{0}=\delta\left(t_{0}, \varepsilon\right), T\left(t_{0}, \varepsilon\right)>0$ such that $\|\varphi\|<\delta_{0}$ implies $|x(t)| \leq \varepsilon$, for $t \geq t_{0}+T\left(t_{0}, \varepsilon\right)$; that is, $\lim _{t \rightarrow+\infty} x(t)=0$. It is uniformly stable if it is stable and $\delta=$ $\delta(\varepsilon)>0$ can be chosen independently of $t_{0}$. It is uniformly asymptotically stable if it is uniformly stable and there exists a $\delta_{0}>0$; for any $\eta>0$, there exists a $T=T(\eta)>0$ such that $\|\varphi\|<\delta_{0}$ implies $|x(t)|<\eta$ for $t>t_{0}+T$.

Next, we will address the main core of the paper, the stability of the nonlinear fractional neutral systems (17). Firstly, we consider the case $\mathscr{D}(t, \phi)=\mathscr{D}(\phi)$; that is, $\mathscr{D}$ is independent of $t$. Now, we give the following LyapunovKrasovskii theorems for nonlinear fractional neutral systems (17).

Theorem 12. Let $\mathscr{D}\left(t, x_{t}\right)=x(t)-B x(t-r)$, let $B$ be Schur stable, and let $x=0$ be an equilibrium point of system (17). Then the zero solution of system (17) is stable if and only if there exist a functional $V: \mathbb{R} \times \mathscr{C}(M) \rightarrow \mathbb{R}$ and a continuous function $u(s)$ with $u(s)>0$ for $s>0$ and $u(0)=0$ such that the following conditions are satisfied.

(1) $V(t, 0)=0$.

(2) $V(t, \phi) \geq u(|\mathscr{D}(\phi)|)$.

(3) For any given $t_{0}$ the functional $V\left(t_{0}, \phi\right)$ is continuous in $\phi$ at the point 0 ; that is, for any $\varepsilon>0$ there exists $\delta>$ 0 such that the inequality $\|\phi\|<\delta$ implies $\mid V\left(t_{0}, \phi\right)-$ $V\left(t_{0}, 0\right) \mid=V\left(t_{0}, \phi\right)<\varepsilon$.

(4) Along the solutions of system (17) the functional $V(t, \phi)$ satisfies $V\left(t, x_{t}\left(t_{0}, \varphi\right)\right) \leq V\left(t_{0}, \varphi\right)$ for $t \geq t_{0}$.

Proof.

Sufficiency. Since the matrix $B$ is Schur stable, there exist $L \geq 1$ and $0<\zeta<1$ such that the inequality $\left\|B^{k}\right\|_{0} \leq L \zeta^{k}$ holds for $k \geq 0$.

For a given $\varepsilon>0(\varepsilon<M)$, we set $\varepsilon_{1}=\varepsilon(1-\zeta) / L>0$. Since for a given $t_{0}$ functional $V\left(t_{0}, \phi\right)$ is continuous in $\phi$ at the point 0 , there exists $\delta_{1}\left(\varepsilon, t_{0}\right)$ such that $V\left(t_{0}, \phi\right)<u\left(\varepsilon_{1}\right)$ 
for any $\phi \in \mathscr{C}$, with $\|\phi\|<\delta_{1}\left(\varepsilon, t_{0}\right)$. Here, we claim that $\delta_{1}\left(\varepsilon, t_{0}\right) \leq \varepsilon_{1}$. Then, there exists an initial function $\varphi \in \mathscr{C}$ such that $\|\varphi\|<\delta_{1}\left(\varepsilon, t_{0}\right)$ and $|\mathscr{D}(\varphi)|=\varepsilon_{1}$. On the one hand, for this initial function, we have $u(|\mathscr{D}(\varphi)|)=u\left(\varepsilon_{1}\right)$. On the other hand, $u(|\mathscr{D}(\varphi)|) \leq V\left(t_{0}, \varphi\right)<u\left(\varepsilon_{1}\right)$. The contradiction proves the desired inequality.

Now we take $\delta\left(\varepsilon, t_{0}\right)=\delta_{1}\left(\varepsilon, t_{0}\right) /(1+L \zeta)$. For $\varphi \in \mathscr{C}$ with $\|\varphi\|<\delta\left(\varepsilon, t_{0}\right)$, we have

$$
\begin{aligned}
u\left(\left|\mathscr{D}\left(x_{t}\left(t_{0}, \varphi\right)\right)\right|\right) & \leq V\left(t, x_{t}\left(t_{0}, \varphi\right)\right) \leq V\left(t_{0}, \varphi\right) \\
& <u\left(\varepsilon_{1}\right), \quad t \geq t_{0} .
\end{aligned}
$$

Next, we wish to show

$$
\left|\mathscr{D}\left(x_{t}\left(t_{0}, \varphi\right)\right)\right|<\varepsilon_{1}, \quad t \geq t_{0} .
$$

Assume by contradiction that there exists a $t_{1} \geq t_{0}$ for which

$$
\left|\mathscr{D}\left(x_{t_{1}}\left(t_{0}, \varphi\right)\right)\right| \geq \varepsilon_{1} .
$$

Since

$$
\begin{aligned}
\left|\mathscr{D}\left(x_{t_{0}}\left(t_{0}, \varphi\right)\right)\right| & =|\mathscr{D}(\varphi)| \leq(1+L \zeta)\|\varphi\| \\
& <\delta_{1}\left(\varepsilon, t_{0}\right) \leq \varepsilon_{1},
\end{aligned}
$$

and $\left|\mathscr{D}\left(x_{t}\left(t_{0}, \varphi\right)\right)\right|$ is a continuous function of $t$, there exists $\bar{t} \in\left[t_{0}, t_{1}\right]$ such that

$$
\left|\mathscr{D}\left(x_{\bar{t}}\left(t_{0}, \varphi\right)\right)\right|=\varepsilon_{1} .
$$

On the one hand, we have

$$
u\left(\left|\mathscr{D}\left(x_{\bar{t}}\left(t_{0}, \varphi\right)\right)\right|\right)=u\left(\varepsilon_{1}\right) .
$$

On the other hand, relation (20) provides the following inequality:

$$
u\left(\left|\mathscr{D}\left(x_{\bar{t}}\left(t_{0}, \varphi\right)\right)\right|\right)<u\left(\varepsilon_{1}\right) .
$$

The contradiction proves that inequality (22) is wrong and relation (21) is true. Then there exists a function $z(t)$ with $|z(t)|<\varepsilon_{1}, t \geq t_{0}$ such that

$$
x\left(t, t_{0}, \varphi\right)=B x\left(t-r, t_{0}, \varphi\right)+z(t), \quad t \geq t_{0} .
$$

For a given $t \geq t_{0}$, there must exist a positive integer number $k$ such that $t \in\left[t_{0}+(k-1) r, t_{0}+k r\right]$. Iterating equality (27) $k-1$ times we obtain

$$
x\left(t, t_{0}, \varphi\right)=B^{k} x\left(t-k r, t_{0}, \varphi\right)+\sum_{i=0}^{k-1} B^{i} z(t-i r) .
$$

Since $t-k r \in\left[t_{0}-r, t_{0}\right]$,

$$
\left|x\left(t-k r, t_{0}, \varphi\right)\right| \leq\|\varphi\|<\delta\left(\varepsilon, t_{0}\right) \leq \varepsilon_{1},
$$

and we obtain the following inequality:

$$
\begin{aligned}
\left|x\left(t, t_{0}, \varphi\right)\right| \leq & \left\|B^{k}\right\|_{0}\left|x\left(t-k r, t_{0}, \varphi\right)\right| \\
& +\sum_{i=0}^{k-1}\left\|B^{i}\right\|_{0}|z(t-i r)| \\
< & L \zeta^{k} \delta\left(\varepsilon, t_{0}\right)+\sum_{i=0}^{k-1} L \zeta^{i} \varepsilon_{1}<\frac{L}{1-\zeta^{1}} \varepsilon_{1} \\
= & \varepsilon, \quad t \geq t_{0} .
\end{aligned}
$$

Therefore, the zero solution of system (17) is stable.
Necessity. Now, the zero solution of system (17) is stable, and we must prove that there exist a function $u(s)$ and a functional $V(t, \phi)$ that satisfy the conditions (1)-(4).

Since the zero solution of system (17) is stable, for $\varepsilon=M$ there exists $\delta\left(M, t_{0}\right)$ such that the inequality $\|\varphi\|<\delta\left(M, t_{0}\right)$ implies that $\left.\mid x\left(t, t_{0}, \varphi\right)\right) \mid<M$ for $t \geq t_{0}$. We define the function $u(s)=s, s \in \mathbb{R}$ and the functional $V(t, \phi)$ as follows:

$$
\begin{aligned}
V\left(t, x_{t}\left(t_{0}, \varphi\right)\right) \\
=\left\{\begin{array}{l}
\sup _{s \geq t}\left|\mathscr{D}\left(x_{s}\left(t_{0}, \varphi\right)\right)\right|, \\
\quad \text { if }\left|x\left(s, t_{0}, \varphi\right)\right|<M, \text { for } s \geq t_{0}, \\
(1+L \zeta) M, \\
\text { if } \exists T \geq t_{0} \text { such that }\left|x\left(T, t_{0}, \varphi\right)\right| \geq M .
\end{array}\right.
\end{aligned}
$$

Since for $\varphi=0$ the corresponding solution is trivial, $x\left(t, t_{0}, 0\right)=0, t \geq t_{0}$, we obtain $V(t, 0)=0$. In the case where $\left|x\left(s, t_{0}, \varphi\right)\right|<M, s \geq t_{0}$, we have

$$
\begin{aligned}
u\left(\left|\mathscr{D}\left(x_{t}\left(t_{0}, \varphi\right)\right)\right|\right) & =\left|\mathscr{D}\left(x_{t}\left(t_{0}, \varphi\right)\right)\right| \\
& \leq \sup _{s \geq t}\left|\mathscr{D}\left(x_{s}\left(t_{0}, \varphi\right)\right)\right|=V\left(t, x_{t}\left(t_{0}, \varphi\right)\right) .
\end{aligned}
$$

In the other case where there exists $T \geq t_{0}$ such that $\left|x\left(T, t_{0}, \varphi\right)\right| \geq M$, the following inequality holds:

$$
\begin{aligned}
u\left(\left|\mathscr{D}\left(x_{t}\left(t_{0}, \varphi\right)\right)\right|\right) & =\left|\mathscr{D}\left(x_{t}\left(t_{0}, \varphi\right)\right)\right| \\
& \leq(1+L \zeta) M=V\left(t, x_{t}\left(t_{0}, \varphi\right)\right) .
\end{aligned}
$$

Further, for a given $t_{0}$, the stability of the zero solution means that for any $\varepsilon>0$ there exists $\delta_{1}=\delta\left(\varepsilon /(1+L \zeta), t_{0}\right)$ such that $\|\varphi\| \leq \delta_{1}$ implies

$$
\left|x\left(t, t_{0}, \varphi\right)\right|<\frac{\varepsilon}{1+L \zeta}, \quad t \geq t_{0} .
$$

Then

$$
\begin{aligned}
\left|\mathscr{D}\left(x_{t}\left(t_{0}, \varphi\right)\right)\right| & \leq\left|x\left(t, t_{0}, \varphi\right)\right|+L \zeta\left|x\left(t-r, t_{0}, \varphi\right)\right| \\
& <\varepsilon, \quad t \geq t_{0} .
\end{aligned}
$$

Therefore,

$$
\left|V\left(t_{0}, \varphi\right)-V\left(t_{0}, 0\right)\right|=V\left(t_{0}, 0\right) \leq \varepsilon .
$$

That is, for a fixed $t_{0}$ the functional $V\left(t_{0}, \varphi\right)$ is continuous in $\varphi$ at the point 0 .

Finally, we need to show $V\left(t, x_{t}\left(t_{0}, \varphi\right)\right) \leq V\left(t_{0}, \varphi\right), t \geq t_{0}$. First, if $\left|x\left(s, t_{0}, \varphi\right)\right|<M$ for $s \geq t_{0}$,

$$
\begin{gathered}
V\left(t_{0}, \varphi\right)=\sup _{s \geq t_{0}}\left|\mathscr{D}\left(x_{s}\left(t_{0}, \varphi\right)\right)\right|, \\
V\left(t, x_{t}\left(t_{0}, \varphi\right)\right)=\sup _{s \geq t}\left|\mathscr{D}\left(x_{s}\left(t_{0}, \varphi\right)\right)\right| .
\end{gathered}
$$

Note that $\{s: s \geq t\} \subseteq\left\{s: s \geq t_{0}\right\}$ for $t \geq t_{0}$; then we have

$$
V\left(t, x_{t}\left(t_{0}, \varphi\right)\right) \leq V\left(t_{0}, \varphi\right) .
$$


In the second case, there exists $T \geq t_{0}$ such that $\left|x\left(T, t_{0}, \varphi\right)\right| \geq$ $M$; we have

$$
V\left(t, x_{t}\left(t_{0}, \varphi\right)\right)=V\left(t_{0}, \varphi\right)=(1+L \zeta) M
$$

Therefore, we have

$$
V\left(t, x_{t}\left(t_{0}, \varphi\right)\right) \leq V\left(t_{0}, \varphi\right), \quad t \geq t_{0} .
$$

Then, the proof is complete.

Remark 13. The functional (31) has only an academic value. Obviously, we cannot use such functionals in applications. The computation of practically useful Lyapunov functionals is a very difficult task.

Theorem 14. Let $\mathscr{D}\left(t, x_{t}\right)=x(t)-B x(t-r), B$ be Schur stable and let $x=0$ be an equilibrium point of system (17). Suppose $u(s)$ is a continuous function with $u(s)>0$ for $s>0$ and $u(0)=$ 0 . If there exists a continuous functional $V: \mathbb{R} \times \mathscr{C}(M) \rightarrow \mathbb{R}$ such that the following conditions are satisfied:

(1) $V(t, 0)=0$,

(2) $V(t, \phi) \geq u(|\mathscr{D}(\phi)|)$,

(3) along the solutions of the system (5) the functional $V(t, \phi)$ is continuously differentiable and satisfies

$$
{ }^{c} D_{t_{0}}^{\beta} V\left(t, x_{t}\left(t_{0}, \varphi\right)\right) \leq 0, \quad t \geq t_{0},
$$

where $\beta \in(0,1]$,

then the zero solution of system (17) is stable.

Proof. Note that the theorem conditions imply that of Theorem 12; therefore, the zero solution of system (17) is stable.

Theorem 15. Suppose that the assumptions in Theorem 14 are satisfied except replacing ${ }^{c} D_{t_{0}}^{\beta}$ by $D_{t_{0}}^{\beta}$; then one has the same result for stability.

Proof. By using Property 4 we have

$$
{ }^{c} D_{t_{0}}^{\beta} V\left(t, x_{t}\right)=D_{t_{0}}^{\beta} V\left(t, x_{t}\right)-V\left(t_{0}, \varphi\right) \frac{\left(t-t_{0}\right)^{-\beta}}{\Gamma(1-\beta)} .
$$

Since $V\left(t_{0}, \varphi\right) \geq 0$, then ${ }^{c} D_{t_{0}}^{\beta} V\left(t, x_{t}\right) \leq D_{t_{0}}^{\beta} V\left(t, x_{t}\right)$. Then we can obtain the same result for stability.

Next, we present the following sufficient conditions for the asymptotic stability of the zero solution of system (17).

Theorem 16. Let $\mathscr{D}\left(t, x_{t}\right)=x(t)-B x(t-r)$, let $B$ be Schur stable, and let $x=0$ be an equilibrium point of system (17). Suppose $u(s), w(s)$ are continuous functions, $u(s), w(s)$ are positive for $s>0$, and $u(0)=w(0)=0$. If there exists $a$ continuously differentiable functional $V: \mathbb{R} \times \mathscr{C}(M) \rightarrow \mathbb{R}$ such that the following conditions are satisfied:

(1) $V(t, 0)=0$,

(2) $V(t, \phi) \geq u(|\mathscr{D}(\phi)|)$,

(3) ${ }^{c} D_{t_{0}}^{\beta} V\left(t, x_{t}\left(t_{0}, \varphi\right)\right) \leq-w\left(\left|\mathscr{D}\left(x_{t}\left(t_{0}, \varphi\right)\right)\right|\right)$, where $\beta \epsilon$ $(0,1]$,

then the zero solution of system (17) is asymptotically stable.

Proof. Since the matrix $B$ is Schur stable, there exist $L \geq 1$ and $0<\zeta<1$ such that the inequality $\left\|B^{k}\right\|_{0} \leq L \zeta^{k}$ holds for $k \geq 0$.

Note that the conditions (1)-(3) of the theorem imply that of Theorem 14; then the zero solution of system (17) is stable; that is, for any $\varepsilon>0$ and $t_{0}$, there exists $\delta\left(\varepsilon, t_{0}\right)>0$ such that for every initial function $\varphi \in \mathscr{C}$, with $\|\varphi\|<\delta\left(\varepsilon, t_{0}\right)$, the following inequality holds:

$$
\left|x\left(t, t_{0}, \varphi\right)\right|<\varepsilon, \quad t \geq t_{0} .
$$

Now, let $\widetilde{\delta}\left(\varepsilon, t_{0}\right)=\delta\left(\varepsilon /(1+L \zeta), t_{0}\right)>0$. Given $t_{0}$ and an initial function $\varphi \in \mathscr{C}$ with $\|\varphi\|<\widetilde{\delta}\left(\varepsilon, t_{0}\right)$, we have

$$
\begin{aligned}
& \left|x\left(t, t_{0}, \varphi\right)\right|<\frac{\varepsilon}{1+L \zeta}, \quad t \geq t_{0}, \\
& \left|\mathscr{D}\left(x_{t}\left(t_{0}, \varphi\right)\right)\right|<\varepsilon, \quad t \geq t_{0}+r .
\end{aligned}
$$

Next, we wish to show

$$
\begin{aligned}
\lim _{t \rightarrow \infty} \mathscr{D}\left(x_{t}\left(t_{0}, \varphi\right)\right) \\
\quad=\lim _{t \rightarrow \infty}\left[x\left(t, t_{0}, \varphi\right)-B x\left(t-r, t_{0}, \varphi\right)\right]=0 .
\end{aligned}
$$

Suppose not, then there exist $\varepsilon_{0}>0$ and a sequence $\left\{t_{k}\right\}_{k=1}^{\infty}$, $t_{k} \rightarrow \infty$, as $k \rightarrow \infty$ such that

$$
\begin{aligned}
\left|\mathscr{D}\left(x_{t_{k}}\left(t_{0}, \varphi\right)\right)\right| & =\left|x\left(t_{k}, t_{0}, \varphi\right)-B x\left(t_{k}-r, t_{0}, \varphi\right)\right| \\
& \geq \varepsilon_{0}, \quad k \geq 1 .
\end{aligned}
$$

Without loss of generality we may assume that $t_{k+1}-t_{k} \geq r$ for $k \geq 0$. It follows from system (17) that

$$
\begin{gathered}
\mathscr{D}\left(x_{t}\left(t_{0}, \varphi\right)\right)=\mathscr{D}(\varphi)+I_{t_{0}}^{\alpha} f\left(t, x_{t}\left(t_{0}, \varphi\right)\right), \quad t \geq t_{0}, \\
\mathscr{D}\left(x_{t_{k}}\left(t_{0}, \varphi\right)\right)=\mathscr{D}(\varphi)+I_{t_{0}}^{\alpha} f\left(t_{k}, x_{t_{k}}\left(t_{0}, \varphi\right)\right) .
\end{gathered}
$$

Then, we have

$$
\begin{aligned}
\mathscr{D}\left(x_{t}\left(t_{0}, \varphi\right)\right)= & \mathscr{D}\left(x_{t_{k}}\left(t_{0}, \varphi\right)\right)+I_{t_{0}}^{\alpha} f\left(t, x_{t}\left(t_{0}, \varphi\right)\right) \\
& -I_{t_{0}}^{\alpha} f\left(t_{k}, x_{t_{k}}\left(t_{0}, \varphi\right)\right) .
\end{aligned}
$$

Since $f$ takes bounded sets into bounded sets, there is a constant $L_{0}$ such that $\left|f\left(t, x_{t}\left(t_{0}, \varphi\right)\right)\right| \leq L_{0}$ for $t \geq t_{0},\|\varphi\| \leq$ $\widetilde{\delta}\left(\varepsilon, t_{0}\right)$. Then

$$
\left|\mathscr{D}\left(x_{t}\left(t_{0}, \varphi\right)\right)\right| \geq \varepsilon_{0}-\frac{2 L_{0}\left(t-t_{k}\right)^{\alpha}}{\Gamma(1+\alpha)}, \quad t \geq t_{k} .
$$


Hence, for any $k \geq 1$, we have

$$
\left|\mathscr{D}\left(x_{t}\left(t_{0}, \varphi\right)\right)\right| \geq \frac{\varepsilon_{0}}{2}, \quad t \in\left[t_{k}, t_{k}+\theta\right],
$$

where $\theta=\min \left\{r,\left[\Gamma(1+\alpha) \varepsilon_{0} / 4 L_{0}\right]^{1 / \alpha}\right\}$. Let $\xi=$ $\min _{\mathcal{E}_{0} / 2 \leq s \leq \varepsilon} w(s)>0$. Then there exists some $t_{n} \in\left\{t_{k}\right\}_{k=1}^{\infty}$ such that

$$
V\left(t_{0}, \varphi\right)<\frac{\xi}{\Gamma(1+\beta)}\left(t_{n}-t_{0}\right)^{\beta} .
$$

From the third condition of the theorem, we have

$$
{ }^{c} D_{t_{0}}^{\beta} V\left(t_{n}, x_{t_{n}}\left(t_{0}, \varphi\right)\right) \leq-w\left(\left|\mathscr{D}\left(x_{t_{n}}\left(t_{0}, \varphi\right)\right)\right|\right) \leq-\xi .
$$

That is,

$$
V\left(t_{n}, x_{t_{n}}\left(t_{0}, \varphi\right)\right) \leq V\left(t_{0}, \varphi\right)-\frac{\xi}{\Gamma(1+\beta)}\left(t_{n}-t_{0}\right)^{\beta}<0,
$$

which contradicts the condition (2) of the theorem. Therefore, relation (45) is true. This means that there exists a function $z(t)$ with $\lim _{t \rightarrow \infty}|z(t)|=0$ such that

$$
x\left(t, t_{0}, \varphi\right)=B x\left(t-r, t_{0}, \varphi\right)+z(t), \quad t \geq t_{0} .
$$

And given a positive value $\varepsilon_{1}<\varepsilon$, there exists $t_{1}>t_{0}$ such that

$$
|z(t)| \leq \frac{1-\zeta}{2 L} \varepsilon_{1}, \quad t \geq t_{1}
$$

Then there exists $k_{0}$ such that $L \zeta^{k} \varepsilon<(1 / 2) \varepsilon_{1}$ for $k \geq k_{0}$. Thus, we have

$$
\begin{aligned}
x\left(t, t_{0}, \varphi\right)= & B^{k_{0}} x\left(t-k_{0} r, t_{0}, \varphi\right) \\
& +\sum_{i=0}^{k_{0}-1} B^{i} z(t-i r), \quad t \geq t_{1}+k_{0} r, \\
\left|x\left(t, t_{0}, \varphi\right)\right| \leq & \left\|B^{k_{0}}\right\|_{0}\left|x\left(t-k_{0} r, t_{0}, \varphi\right)\right| \\
& +\sum_{i=0}^{k_{0}-1}\left\|B^{i}\right\|_{0}|z(t-i r)| \\
< & L \zeta^{k_{0}} \varepsilon+\frac{\varepsilon_{1}}{2}<\varepsilon_{1}, \quad t \geq t_{1}+k_{0} r .
\end{aligned}
$$

Therefore, we have $\lim _{t \rightarrow \infty} x\left(t, t_{0}, \varphi\right)=0$; that is, the zero solution of system (17) is asymptotically stable.

Theorem 17. Suppose that the assumptions in Theorem 16 are satisfied except replacing ${ }^{c} D_{t_{0}}^{\beta}$ by $D_{t_{0}}^{\beta}$; then one has the same result for asymptotical stability.

Proof. By using Property 4 we have

$$
{ }^{c} D_{t_{0}}^{\beta} V\left(t, x_{t}\right)=D_{t_{0}}^{\beta} V\left(t, x_{t}\right)-V\left(t_{0}, \varphi\right) \frac{\left(t-t_{0}\right)^{-\beta}}{\Gamma(1-\beta)} .
$$

Since $V\left(t_{0}, \varphi\right) \geq 0$, then ${ }^{c} D_{t_{0}}^{\beta} V\left(t, x_{t}\right) \leq D_{t_{0}}^{\beta} V\left(t, x_{t}\right)$. Then we can obtain the same result for asymptotical stability.
Here, we present the following sufficient conditions for the instability of the zero solution of system (17).

Theorem 18. Suppose $\mathscr{D}$ in (17) is independent of $t, V: \mathscr{C} \rightarrow$ $\mathbb{R}$ is continuous and maps bounded sets into bounded sets, and there exist continuous, nondecreasing nonnegative functions $a(s), b(s), s \geq 0$, positive for $s>0$, an open set $S$ in $\mathscr{C}$, and a bounded open neighborhood $U$ of zero in $\mathscr{C}$ such that $V(\phi)$ satisfies the following.

(i) $V(\phi)>0$ on $S, V(\phi)=0$ on the boundary $\partial S$ of $S$.

(ii) 0 belongs to the closure of $S \cap U$.

(iii) $V(\phi) \leq a(|\mathscr{D}(\phi)|)$ on $\mathbb{R} \times(S \cap U)$.

(iv) ${ }^{c} D_{t_{0}}^{\beta} V(\phi) \geq b(|\mathscr{D}(\phi)|)$ on $\mathbb{R} \times(S \cap U), \beta \in(0,1]$.

Under these conditions, the zero solution of (17) is unstable. More specifically, each solution $x=x\left(t, t_{0}, \varphi\right)$ of (17) with initial value $\varphi$ in $S \cap U$ at $t_{0} \in \mathbb{R}$ must reach the boundary of $U$ in finite time.

Proof. Suppose $\varphi_{0} \in S \cap U, t_{0} \in \mathbb{R}$. Then $V\left(\varphi_{0}\right)>0$. From (iv), the solution $x\left(t, t_{0}, \varphi_{0}\right)$ satisfies $V\left(x_{t}\right) \geq V\left(\varphi_{0}\right)$ as long as $x_{t} \in S \cap U$. From (iii) and (iv), this implies

$$
\begin{aligned}
{ }^{c} D_{t_{0}}^{\beta} V\left(x_{t}\right) & \geq b\left(\left|\mathscr{D}\left(x_{t}\right)\right|\right) \geq b\left(a^{-1}\left(V\left(x_{t}\right)\right)\right) \\
& \geq b\left(a^{-1}\left(V\left(\varphi_{0}\right)\right)\right)>0,
\end{aligned}
$$

as long as $x_{t} \in S \cap U$. Then this relation implies

$$
V\left(x_{t}\right) \geq V\left(\varphi_{0}\right)+b\left(a^{-1}\left(V\left(\varphi_{0}\right)\right)\right) \frac{\left(t-t_{0}\right)^{\beta}}{\Gamma(1+\beta)},
$$

as long as $x_{t} \in S \cap U$. Since $U$ is bounded and $V$ is bounded on $U$, there must be a $t_{1}$ such that $x_{t_{1}} \in \partial(S \cap U)$. But hypothesis (i) implies that $x_{t_{1}} \in \partial(U)$. This proves the last assertion of the theorem. Hypothesis (ii) implies that each neighborhood of zero contains a $\varphi_{0}$ in $S \cap U$. Thus, $x=0$ is unstable and the theorem is proved.

Finally, we consider that the case $\mathscr{D}$ is dependent of $t$ and give the following Lyapunov-Krasovskii theorems for nonlinear fractional neutral systems (17).

Theorem 19. Suppose $\mathscr{D}$ is uniformly stable with respect to $C\left(\mathbb{R}, \mathbb{R}^{n}\right), u(s), v(s), w(s)$ are continuous nondecreasing functions, $u(s), v(s)$ are positive for $s>0, u(0)=v(0)=0$, and $w(s)$ is nonnegative. If there exists a continuously differentiable functional $V: \mathbb{R} \times \mathscr{C} \rightarrow \mathbb{R}$ such that

(i) $u(|\mathscr{D}(t, \phi)|) \leq V(t, \phi) \leq v(\|\phi\|)$,

(ii) ${ }^{c} D_{t_{0}}^{\beta} V\left(t, x_{t}\right) \leq-w\left(\left|\mathscr{D}\left(t, x_{t}\right)\right|\right), \beta \in(0,1]$,

then the zero solution of (17) is uniformly stable. If, in addition, $w(s)>0$ for $s>0$, then it is uniformly asymptotically stable.

Proof. Suppose the constants $b, c, d$ are defined as in Lemma 7, and $x(t)=x\left(t, t_{0}, \varphi\right)$ is solution of (17)-(18). For any $\varepsilon>0$, we can find a sufficiently small $\delta$ such that 
$b \delta<\varepsilon / 2, v(\delta)<u(\varepsilon / 2(c+d))$. Hence, for any initial time $t_{0}$ and any initial condition $x_{t_{0}}=\varphi$ with $\|\varphi\|<\delta$, we have ${ }^{c} D_{t_{0}}^{\beta} V\left(t, x_{t}\left(t_{0}, \varphi\right)\right) \leq 0$, and therefore $V\left(t, x_{t}\left(t_{0}, \varphi\right)\right) \leq$ $V\left(t_{0}, \varphi\right)$ for any $t \geq t_{0}$. This implies that

$$
\begin{aligned}
u\left(\left|\mathscr{D}\left(t, x_{t}\left(t_{0}, \varphi\right)\right)\right|\right) & \leq V\left(t, x_{t}\left(t_{0}, \varphi\right)\right) \leq V\left(t_{0}, \varphi\right) \\
& \leq v(\delta)<u\left(\frac{\varepsilon}{2(c+d)}\right),
\end{aligned}
$$

which implies that $\left|\mathscr{D}\left(t, x_{t}\left(t_{0}, \varphi\right)\right)\right|<\varepsilon / 2(c+d)$ for $t \geq t_{0}$. Since $\mathscr{D}$ is uniformly stable, Lemma 7 implies

$$
\begin{aligned}
\left\|x_{t}\left(t_{0}, \varphi\right)\right\| & \leq b\|\varphi\|+(c+d) \frac{\varepsilon}{2(c+d)} \\
& <b \delta+\frac{\varepsilon}{2}<\varepsilon .
\end{aligned}
$$

Therefore, the zero solution is uniformly stable.

To prove uniform asymptotic stability, let $\varepsilon_{0}:=\varepsilon=1$; choose $\delta_{0}:=\delta(1)>0$ which correspond to uniform stability. Then, for any $t_{0} \in \mathbb{R},\|\varphi\|<\delta_{0}$ implies

$$
\begin{array}{r}
\left\|x_{t}\left(t_{0}, \varphi\right)\right\|<\varepsilon_{0}, \quad\left|\mathscr{D}\left(t, x_{t}\left(t_{0}, \varphi\right)\right)\right|<\frac{\varepsilon_{0}}{2(c+d)}, \\
t \geq t_{0} .
\end{array}
$$

Next, for any $\eta>0$, we wish to show that there is a $T\left(\delta_{0}, \eta\right)$ such that any solution $x\left(t, t_{0}, \varphi\right)$ of $(17)$ with $\|\varphi\|<\delta_{0}$ satisfies $\left\|x_{t}\left(t_{0}, \varphi\right)\right\|<\eta$ for $t \geq t_{0}+T\left(\delta_{0}, \eta\right)$. To do this, we show that there is a $T\left(\delta_{0}, \eta\right)$ and $t_{1}$ in $\left[t_{0}, t_{0}+T\left(\delta_{0}, \eta\right)\right]$ such that $\left\|x_{t_{1}}\left(t_{0}, \varphi\right)\right\|<\delta$, where $\delta=\delta(\eta)$ is the above constant for uniform stability. The uniform stability then implies that $\left\|x_{t}\left(t_{0}, \varphi\right)\right\|<\eta$ for $t \geq t_{1}$ and, in particular, for $t \geq t_{0}+T\left(\delta_{0}, \eta\right)$.

For $a, b, c, d$ as in Lemma 7 , choose $N=N\left(\delta_{0}, \eta\right)$ so that

$$
e^{-a N}\left(b \delta_{0}+\frac{c}{2(c+d)}\right) \leq \frac{\delta}{2} .
$$

Let $T\left(\delta_{0}, \eta\right)=N+r+\left[v\left(\delta_{0}\right) \Gamma(1+\beta) / w(\delta / 2 d)\right]^{1 / \beta}$. Suppose there is a solution $x=x\left(t, t_{0}, \varphi\right)$ of (17) with $\|\varphi\|<\delta_{0}$ and $\left\|x_{t}\left(t_{0}, \varphi\right)\right\| \geq \delta$ for $t \geq t_{0}$. Taking $s=t_{0}+\left[v\left(\delta_{0}\right) \Gamma(1+\right.$ $\beta) / w(\delta / 2 d)]^{1 / \beta}, t=s^{\prime}:=s+N+r$ in relation (12), we have

$$
\begin{aligned}
\delta \leq & \left\|x_{s^{\prime}}\right\| \\
\leq & e^{-a N}\left(b\|\varphi\|+c \sup _{u \in\left[t_{0}, s^{\prime}\right]}\left|\mathscr{D}\left(u, x_{u}\right)\right|\right) \\
& +d \sup _{u \in\left[s, s^{\prime}\right]}\left|\mathscr{D}\left(u, x_{u}\right)\right| \\
\leq & e^{-a N}\left(b \delta_{0}+\frac{c}{2(c+d)}\right)+d \sup _{u \in[s, s+N+r]}\left|\mathscr{D}\left(u, x_{u}\right)\right| \\
\leq & \frac{\delta}{2}+d \sup _{u \in\left[s, s^{\prime}\right]}\left|\mathscr{D}\left(u, x_{u}\right)\right| .
\end{aligned}
$$

Therefore, there exists a $t^{\prime} \in\left[s, s^{\prime}\right]$ such that $\left|\mathscr{D}\left(t^{\prime}, x_{t^{\prime}}\right)\right|>$ $\delta / 2 d$. Then, by the condition (ii) of the theorem, we have

$$
{ }^{c} D_{t_{0}}^{\beta} V\left(t^{\prime}, x_{t^{\prime}}\right) \leq-w\left(\left|\mathscr{D}\left(t^{\prime}, x_{t^{\prime}}\right)\right|\right) \leq-w\left(\frac{\delta}{2 d}\right),
$$

and hence by Property 4 we conclude

$$
{ }^{c} D_{t_{0}}^{\beta}\left[V\left(t^{\prime}, x_{t^{\prime}}\right)+w\left(\frac{\delta}{2 d}\right) \frac{\left(t^{\prime}-t_{0}\right)^{\beta}}{\Gamma(1+\beta)}\right] \leq 0 .
$$

Then by using Property 4 and condition (i) of the theorem, we have

$$
\begin{gathered}
V\left(t^{\prime}, x_{t^{\prime}}\right)+w\left(\frac{\delta}{2 d}\right) \frac{\left(t^{\prime}-t_{0}\right)^{\beta}}{\Gamma(1+\beta)} \\
\leq V\left(t_{0}, \varphi\right) \leq v(\|\phi\|) \leq v\left(\delta_{0}\right) .
\end{gathered}
$$

As a result we obtain

$$
\begin{aligned}
0 & <u\left(\frac{\delta}{2 d}\right) \leq V\left(t^{\prime}, x_{t^{\prime}}\right) \\
& \leq v\left(\delta_{0}\right)-w\left(\frac{\delta}{2 d}\right) \frac{\left(t^{\prime}-t_{0}\right)^{\beta}}{\Gamma(1+\beta)} \\
& \leq v\left(\delta_{0}\right)-w\left(\frac{\delta}{2 d}\right) \frac{\left(s-t_{0}\right)^{\beta}}{\Gamma(1+\beta)}=0 .
\end{aligned}
$$

This contradiction proves that there exists a $t_{1} \epsilon$ $\left[t_{0}, t_{0}+T\left(\delta_{0}, \eta\right)\right]$ such that $\left\|x_{t_{1}}\left(t_{0}, \varphi\right)\right\|<\delta$. Thus, we have $\left|x\left(t, t_{0}, \varphi\right)\right|<\eta, t \geq t_{0}+T\left(\delta_{0}, \eta\right)$, whenever $\|\varphi\|<\delta_{0}$. This proves the uniform asymptotic stability of the zero solution of (17).

Theorem 20. Suppose that the assumptions in Theorem 19 are satisfied except replacing ${ }^{c} D_{t_{0}}^{\beta}$ by $D_{t_{0}}^{\beta}$; then one has the same result for uniform stability and uniform asymptotic stability.

Proof. By using Property 4 we have

$$
{ }^{c} D_{t_{0}}^{\beta} V\left(t, x_{t}\right)=D_{t_{0}}^{\beta} V\left(t, x_{t}\right)-V\left(t_{0}, \varphi\right) \frac{\left(t-t_{0}\right)^{-\beta}}{\Gamma(1-\beta)} .
$$

Since $V\left(t_{0}, \varphi\right) \geq 0$, then ${ }^{c} D_{t_{0}}^{\beta} V\left(t, x_{t}\right) \leq D_{t_{0}}^{\beta} V\left(t, x_{t}\right)$. Then we can obtain the same result for uniform stability and uniform asymptotic stability.

\section{An Illustrative Example}

A fractional neutral differential difference system is considered in the following state-space description:

$$
\begin{aligned}
{ }^{c} D_{t_{0}}^{\alpha}\left[\mathscr{D}\left(x_{t}\right)\right] & =f\left(\mathscr{D}\left(x_{t}\right)\right), \\
x_{t_{0}} & =\varphi,
\end{aligned}
$$

where $0<\alpha<1, \mathscr{D}\left(x_{t}\right)=x(t)-B x(t-r), B$ is Schur stable, $f(0)=0, f(x)(d f(x) / d x) \dot{x} \leq 0$, and there exist a continuous function $u(s)$ with $u(s)>0$ for $s>0$ and $u(0)=0$ such that $u\left(\left|\mathscr{D}\left(x_{t}\right)\right|\right) \leq\left|f\left(\mathscr{D}\left(x_{t}\right)\right)\right|$; then the equilibrium point $x=0$ of system (70) is stable. 
Proof. Let the Lyapunov candidate be $V\left(t, x_{t}\right)=f^{2}\left(\mathscr{D}\left(x_{t}\right)\right)$, then, for $0<\beta<1$, we have

$$
\begin{aligned}
{ }^{c} & D_{t_{0}}^{\beta} V\left(t, x_{t}\right) \\
& =I_{t_{0}}^{1-\beta}\left(\frac{d V\left(t, x_{t}\right)}{d t}\right) \\
& =2 I_{t_{0}}^{1-\beta}\left\{f\left(\mathscr{D}\left(x_{t}\right)\right)\left[\left.\frac{d f(x)}{d x}\right|_{x=\mathscr{D}\left(x_{t}\right)}\right] \frac{d \mathscr{D}\left(x_{t}\right)}{d t}\right\} \\
& =2 I_{t_{0}}^{1-\beta}\left[f\left(\mathscr{D}\left(x_{t}\right)\right) f^{\prime}\left(\mathscr{D}\left(x_{t}\right)\right) \frac{d \mathscr{D}\left(x_{t}\right)}{d t}\right] \leq 0 .
\end{aligned}
$$

Then, it follows from Theorem 14 that the equilibrium point $x=0$ of system (70) is stable.

\section{Conclusions}

In this paper, we have studied the stability of a class of nonlinear fractional neutral differential difference systems. We introduce the Lyapunov-Krasovskii approach for fractional neutral systems, which enrich the knowledge of both the system theory and the fractional calculus. By using Lyapunov-Krasovskii technique, stability and instability criteria are obtained for the nonlinear fractional neutral differential difference systems. Finally, we point out that since the computation of practically useful Lyapunov functionals is a very difficult task, fractional Lyapunov method has its own limitations. In other words, the present paper is only an introduction to the topic, and there remains a lot of work to do.

\section{Conflict of Interests}

The authors declare that there is no conflict of interests regarding the publication of this paper.

\section{Acknowledgments}

This work is supported by the National Natural Science Foundation of China (11371027), the Fundamental Research Funds for the Central Universities (2013HGXJ0226), and the Fund of Anhui University Graduate Academic Innovation Research (10117700004).

\section{References}

[1] A. A. Kilbas, H. M. Srivastava, and J. J. Trujillo, Theory and Applications of Fractional Differential Equations, Elsevier, 2006.

[2] K. V. Miller and B. Ross, An Introduction to the Fractional Calculus and Fractional Differential Equations, Wiley, New York, NY, USA, 1993.

[3] C. A. Monje, Y. Q. Chen, B. M. Vinagre, D. Xue, and V. Feliu, Fractionalorder Systems and Controls: Fundamentals and Applications, Springer, London, UK, 2010.

[4] I. Podlubny, Fractional Differential Equations, Academic Press, San Diego, Calif, USA, 1999.
[5] S. G. Samko, A. A. Kilbas, and O. I. Marichev, FractionalIntegrals and Derivatives: Theory and Applications, Gordon and Breach Science, Yverdon, Switzerland, 1993.

[6] D. Matignon, "Stability results for fractional differential equations with applications to control processing," in In Proceedings of IMACS-SMC, vol. 2, pp. 963-968, Lille, France, 1996.

[7] C. Bonnet and J. R. Partington, "Analysis of fractional delay systems of retarded and neutral type," Automatica, vol. 38, no. 8, pp. 1133-1138, 2002.

[8] Y. Chen and K. L. Moore, "Analytical stability bound for a class of delayed fractional-order dynamic systems," Nonlinear Dynamics, vol. 29, no. 1-4, pp. 191-200, 2002.

[9] W. Deng, C. Li, and J. Lü, "Stability analysis of linear fractional differential system with multiple time delays," Nonlinear Dynamics, vol. 48, no. 4, pp. 409-416, 2007.

[10] M. P. Lazarevic and A. M. Spasic, "Finite-time stability analysis of fractional order time-delay systems: Gronwall's approach," Mathematical and Computer Modelling, vol. 49, no. 3-4, pp. 475481, 2009.

[11] K. Liu and W. Jiang, "Finite-time stability of linear fractional order neutral systems," Mathematica Applicata, vol. 24, no. 4, pp. 724-730, 2011.

[12] J. Sabatier, M. Moze, and C. Farges, "LMI stability conditions for fractional order systems," Computers \& Mathematics with Applications, vol. 59, no. 5, pp. 1594-1609, 2010.

[13] Y. Li, Y. Q. Chen, and I. Podlubny, "Mittag-Leffler stability of fractional order nonlinear dynamic systems," Automatica, vol. 45, no. 8, pp. 1965-1969, 2009.

[14] Y. Li, Y. Q. Chen, and I. Podlubny, "Stability of fractionalorder nonlinear dynamic systems: lyapunov direct method and generalized Mittag-Leffler stability," Computers \& Mathematics with Applications, vol. 59, no. 5, pp. 1810-1821, 2010.

[15] H. Delavari, D. Baleanu, and J. Sadati, "Stability analysis of Caputo fractional-order nonlinear systems revisited," Nonlinear Dynamics, vol. 67, no. 4, pp. 2433-2439, 2012.

[16] D. Baleanu, A. Ranjbar N., S. J. a. Sadati R., T. Abdeljawad, and V. Gejji, "Lyapunov-Krasovskii stability theorem for fractional systems with delay," Romanian Journal of Physics, vol. 56, no. 56, pp. 636-643, 2011.

[17] D. Baleanu, S. J. Sadati, R. Ghaderi, A. Ranjbar, T. Abdeljawad, and F. Jarad, "Razumikhin stability theorem for fractional systems with delay," Abstract and Applied Analysis, vol. 2010, Article ID 124812, 9 pages, 2010.

[18] S. J. Sadati, D. Baleanu, A. Ranjbar, R. Ghaderi, and T. Abdeljawad, "Mittag-Leffler stability theorem for fractional nonlinear systems with delay," Abstract and Applied Analysis, vol. 2010, Article ID 108651, 7 pages, 2010.

[19] C. P. Li and F. R. Zhang, "A survey on the stability of fractional differential equations," European Physical Journal: Special Topics, vol. 193, no. 1, pp. 27-47, 2011.

[20] M. Rivero, S. V. Rogosin, J. A. Tenreiro Machado, and J. J. Trujillo, "Stability of fractional order systems," Mathematical Problems in Engineering, vol. 2013, Article ID 356215, 14 pages, 2013.

[21] M. A. Cruz and J. K. Hale, "Stability of functional differential equations of neutral type," Journal of Differential Equations, vol. 7, pp. 334-355, 1970.

[22] J. K. Hale and S. M. Verduyn Lunel, Introduction to Functional Differential Equations, Springer, New York, NY, USA, 1993.

[23] R. Fleming, G. Grossman, T. Lenker, S. Narayan, and S. Ong, "On Schur D-stable matrices," Linear Algebra and its Applications, vol. 279, no. 1-3, pp. 39-50, 1998. 


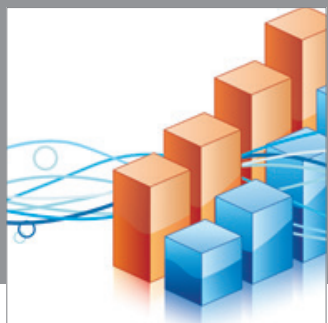

Advances in

Operations Research

mansans

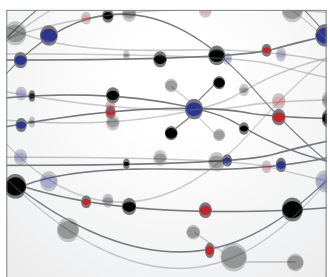

The Scientific World Journal
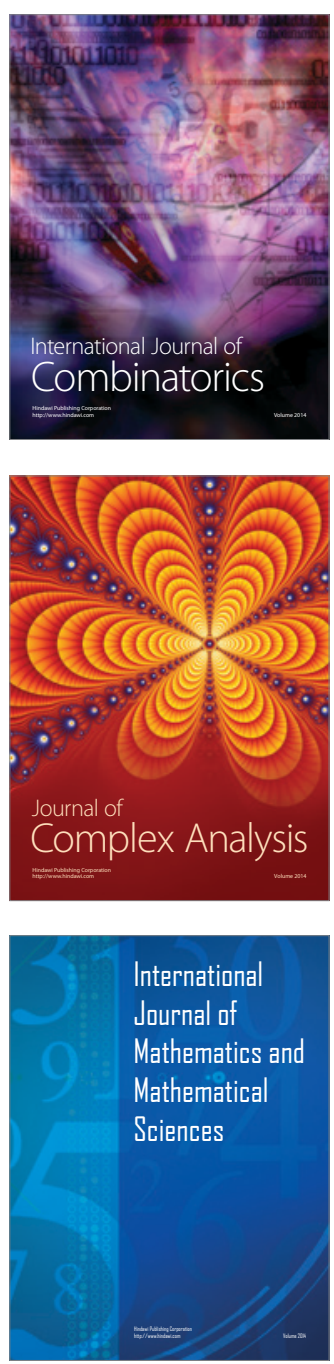
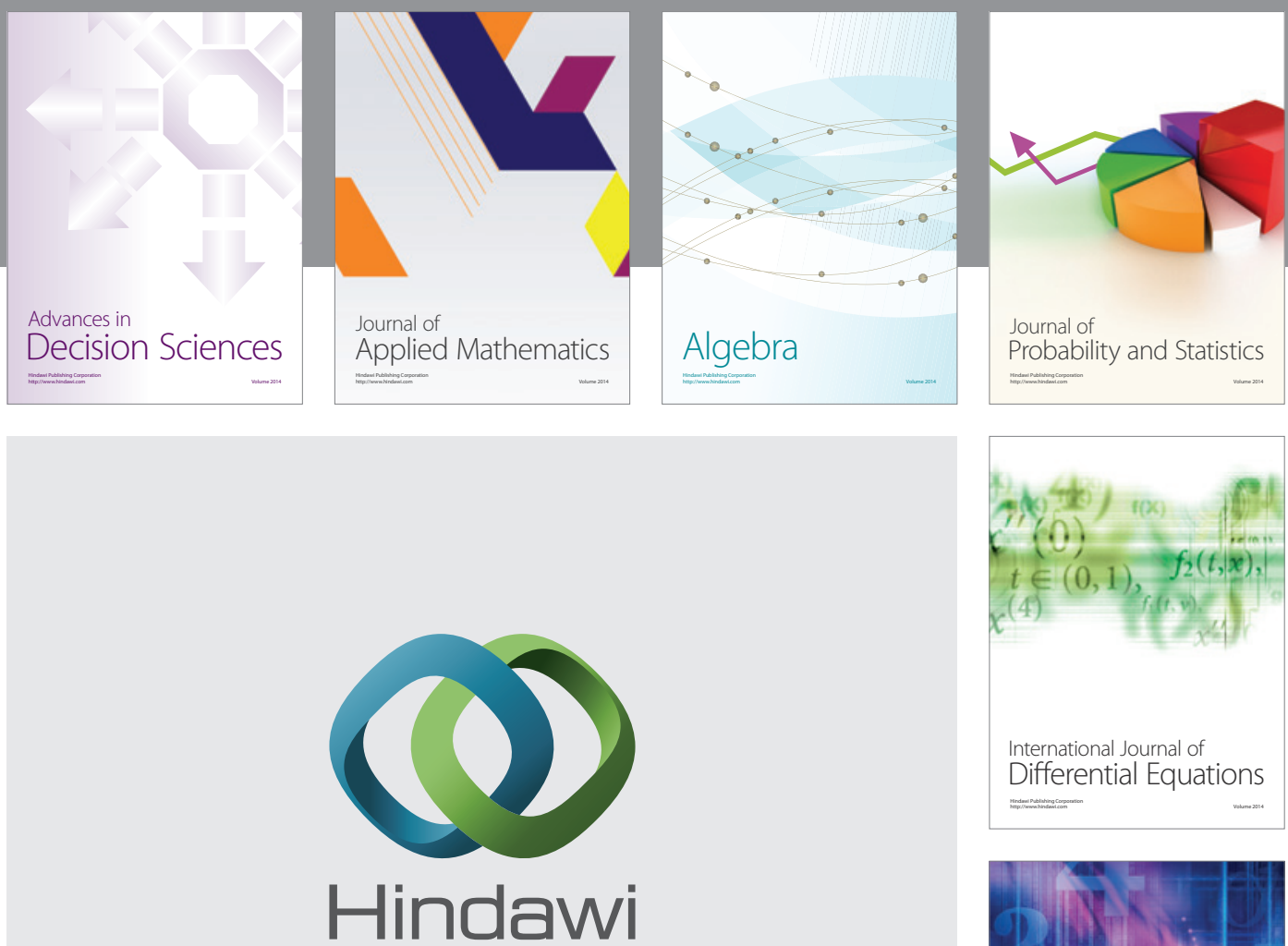

Submit your manuscripts at http://www.hindawi.com
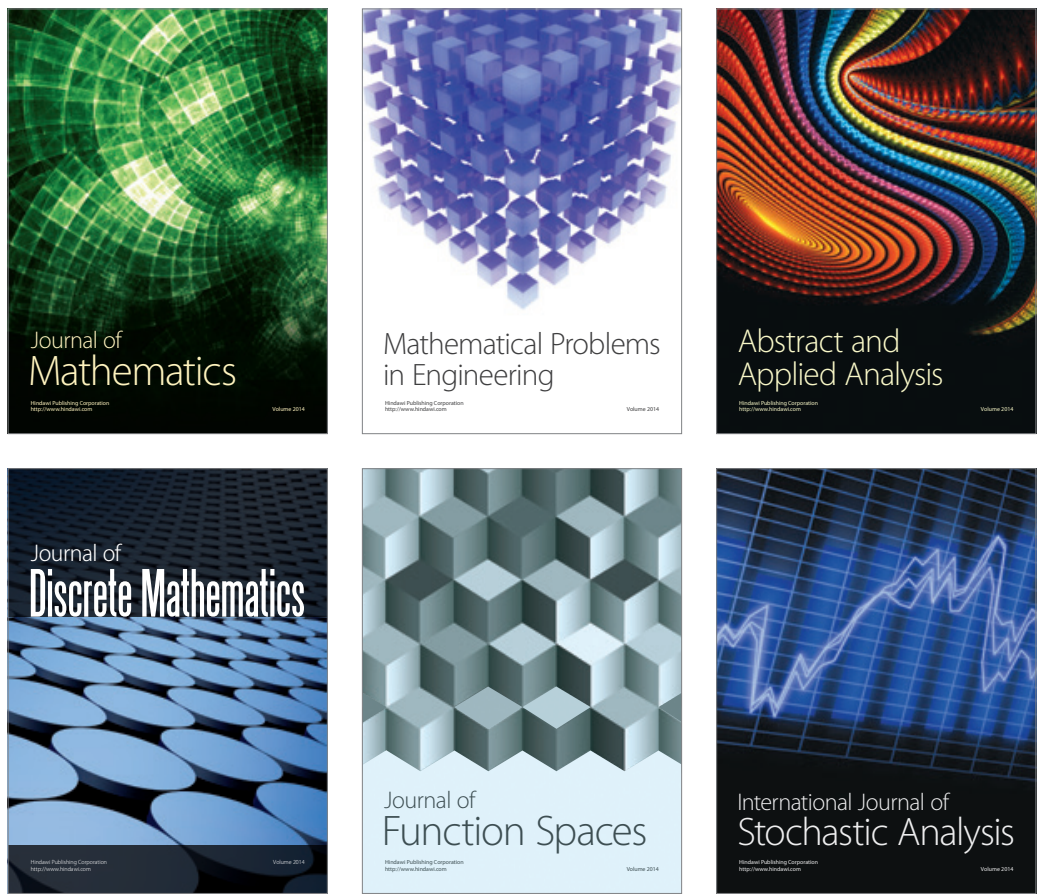

Journal of

Function Spaces

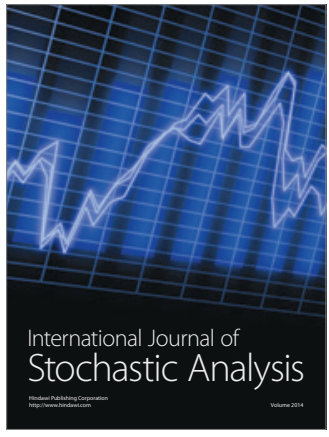

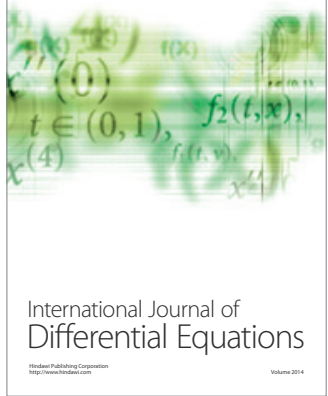
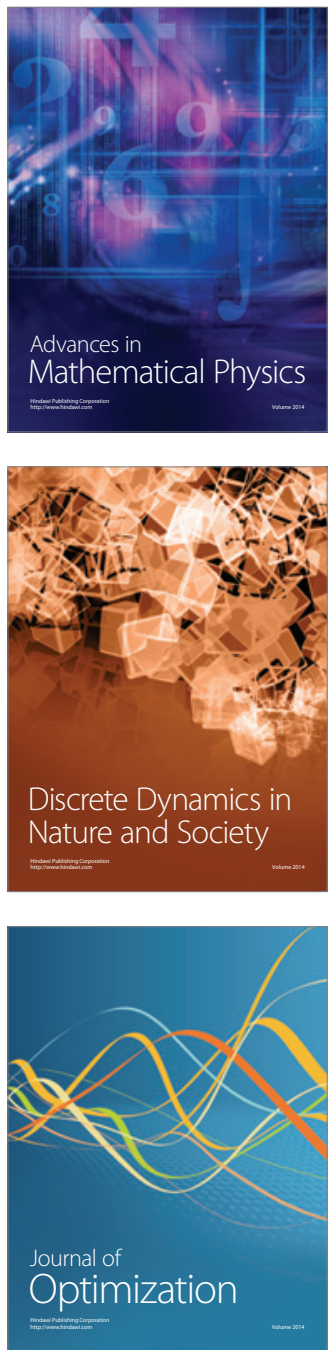